This is a preprint of: "New family of centers of planar polynomial differential systems of arbitrary even degree", Jaume Llibre, Marzieh Mousavi, Arefeh Nabavi, J. Dyn. Control Syst., 12 pages, 2019.

DOI: $[10.1007 / \mathrm{s} 10883-019-09432-\mathrm{x}]$

\title{
NEW FAMILY OF CENTERS OF PLANAR POLYNOMIAL DIFFERENTIAL SYSTEMS OF ARBITRARY EVEN DEGREE
}

\author{
JAUME LLIBRE ${ }^{1}$, MARZIEH MOUSAVI ${ }^{2}$ AND AREFEH NABAVI ${ }^{2}$
}

\begin{abstract}
The problem of distinguish between a focus and a center is one of the classical problems in the qualitative theory of planar differential systems. In this paper we provide a new family of centers of polynomial differential systems of arbitrary even degree. Moreover, we classify the global phase portraits in the Poincaré disc of the centers of this family having degree 2,4 and 6 .
\end{abstract}

\section{Introduction AND STATEMENT OF THE MAIN RESUlts}

Let $P(x, y)$ and $Q(x, y)$ be two real polynomials. In this work we deal with polynomial differential systems in $\mathbb{R}^{2}$ of the form

$$
\dot{x}=P(x, y), \quad \dot{y}=Q(x, y),
$$

where the dot denotes derivative with respect to an independent real variable $t$, usually called the time. The degree of the polynomial differential system (1) is the maximum of the degrees of the polynomials $P(x, y)$ and $Q(x, y)$.

The origin $O=(0,0)$ of $\mathbb{R}^{2}$ is a singular point for system (1) if $P(0,0)=Q(0,0)=0$.

When all the orbits of system (1) in a neighborhood $U \backslash\{O\}$ of the singular point $O$ are periodic, we say that the origin $O$ is a center.

If all the orbits of system (1) in a neighborhood $U \backslash\{O\}$ of the singular point $O$ spiral to $O$ when $t \rightarrow+\infty$ or when $t \rightarrow-\infty$, we say that the origin is a focus.

2010 Mathematics Subject Classification. Primary 34A05. Secondary 34C05, $37 \mathrm{C} 10$.

Key words and phrases. Poincaré compactification, center, first integral, invariant algebraic curve. 
The center-focus problem consists in distinguishing when the singular point $O$ is either a center or a focus. The center-focus problem started with Poincaré [11] and Dulac [1], and in the present days many questions about them remain open. More recent results on the center-focus problem can be found in $[3,4,5,7]$ and in their references.

In this paper we consider the planar polynomial differential systems of the form

(2) $\dot{x}=P(x, y)=y, \quad \dot{y}=Q(x, y)=-x+\prod_{i=1}^{k}\left(x^{2}+y^{2}-r_{i}^{2}\right)$,

of degree $2 k$ depending of $k$ parameters $r_{i}$ for $i=1,2, \ldots, k$ such that $0<r_{1}<r_{2}<\cdots<r_{k}$. We denote the vector field of this system by $\mathrm{X}$.

It is easy to show that the function

$$
V(x, y)=\prod_{i=1}^{k}\left(x^{2}+y^{2}-r_{i}^{2}\right)
$$

satisfies the equality

$$
\frac{\partial V}{\partial x} \dot{x}+\frac{\partial V}{\partial y} \dot{y}=V\left(\frac{\partial P}{\partial x}+\frac{\partial Q}{\partial y}\right) .
$$

Therefore $V$ is an inverse integrating factor of system (2), for more details see for instance [2].

By multiplying the vector field $\mathbf{X}$ by the integrating factor $1 / V$ system (2) becomes a Hamiltonian system. If we compute the Hamiltonian $H$ of that system for $k=1$ we obtain

$$
H(x, y)=e^{-2 x}\left(x^{2}+y^{2}-r_{1}^{2}\right),
$$

for $k=2$ we have

$$
H(x, y)=e^{-2 x\left(r_{1}^{2}-r_{2}^{2}\right)}\left(x^{2}+y^{2}-r_{1}^{2}\right)\left(x^{2}+y^{2}-r_{2}^{2}\right)^{-1},
$$

and for $k=3$ we get

$$
\begin{aligned}
H(x, y)= & e^{-2 x\left(r_{1}^{2}-r_{2}^{2}\right)\left(r_{1}^{2}-r_{3}^{2}\right)\left(r_{2}^{2}-r_{3}^{2}\right)}\left(x^{2}+y^{2}-r_{1}^{2}\right)^{\left(r_{2}^{2}-r_{3}^{2}\right)} \\
& \left(x^{2}+y^{2}-r_{2}^{2}\right)^{\left(r_{3}^{2}-r_{1}^{2}\right)}\left(x^{2}+y^{2}-r_{3}^{2}\right)^{\left(r_{1}^{2}-r_{2}^{2}\right)} .
\end{aligned}
$$

An important property of systems (2), which will help for charaterizing their phase portraits, is that all the circles $f_{i}(x, y)=x^{2}+y^{2}-r_{i}^{2}=0$ for $i=1,2, \ldots, k$ are invariant algebraic curves of system (2), i.e. they are formed by orbits of systems (2), because they satisfy that

$$
\mathbf{X} f_{i}=K_{i} f_{i},
$$


where $K_{i}$ is the polynomial $2 y \prod_{j=1, j \neq i}^{k}\left(x^{2}+y^{2}-r_{j}^{2}\right)$, see Chapter 8 of [2] for additional information on the invariant algebraic curves.

In this paper we prove that polynomial differential systems (2) provide a new family of centers of degree $2 k$ for all $k=1,2, \ldots$ Moreover, we classify the global phase portraits of systems (2) in the Poincaré disc for $k=1,2,3$.

Theorem 1. For $k=1,2, \ldots$ the polynomial differential systems (2) have a unique singular point in the interior of the circle $x^{2}+y^{2}=r_{1}^{2}$ and this singular point is a center.

Theorem 1 is proved in section 3 .

Theorem 2. For $k=1$ the polynomial differential systems (2) have a phase portrait in the Poincaré disc topologically equivalent to the phase portrait of Figure 1.

Theorem 3. For $k=2$ the polynomial differential systems (2) have a phase portrait in the Poincaré disc topologically equivalent to one of the three phase portraits of Figure 3.

Theorem 4. For $k=3$ the polynomial differential systems (2) have a phase portrait in the Poincaré disc topologically equivalent to one of the seven phase portraits of Figure 5.

Theorems 2, 3 and 4 are proved in section 4 .

In section 2 we recall basic definitions and results for proving our theorems.

\section{Preliminary Results}

2.1. Poincaré compactification. In this section we summarize some basic results about the Poincaré compactification, which was done by Poincaré in [11]. He provided a tool for studying the behaviour of a planar polynomial differential system near the infinity. For more details on the Poincaré compactification, see Chapter 5 of [2].

Let $\mathbf{X}=P \frac{\partial}{\partial x_{1}}+Q \frac{\partial}{\partial x_{2}}$ be a polynomial vector field of degree $d$. We consider the Poincaré sphere $\mathbb{S}^{2}=\left\{y=\left(y_{1}, y_{2}, y_{3}\right) \in \mathbb{R}^{3}: y_{1}^{2}+y_{2}^{2}+y_{3}^{2}=\right.$ 1 \}, its tangent plane to the point $(0,0,1)$ is identified with $\mathbb{R}^{2}$. Now we consider the central projection $f: \mathbb{R}^{2} \rightarrow \mathbb{S}^{2}$ of the vector field $\mathbf{X}$, which sends every point $x \in \mathbb{R}^{2}$ to the two intersection points of the straight line passing through the point $x$ and the origin of coordinates 
with the sphere $\mathbb{S}^{2}$. We note that the equator $\mathbb{S}^{1}=\left\{y \in \mathbb{S}^{2}: y_{3}=0\right\}$ of the sphere is in bijection with the infinity of $\mathbb{R}^{2}$. The differential $D f$ sends the vector field $\mathbf{X}$ on $\mathbb{R}^{2}$ into a vector field $\mathbf{X}^{\prime}$ defined on $\mathbb{S}^{2} \backslash \mathbb{S}^{1}$, which is formed by two symmetric copies of $\mathbf{X}$ with respect to the origin of coordinates.

We can extend the vector field $\mathbf{X}^{\prime}$ analytically to a vector field on $\mathbb{S}^{2}$ multiplying $\mathbf{X}^{\prime}$ by $y_{3}^{d}$. This new vector field is denoted by $p(\mathbf{X})$ and it is called the Poincaré compactification of the polynomial vector field $\mathbf{X}$ on $\mathbb{R}^{2}$. The dynamics of $p(\mathbf{X})$ near $\mathbb{S}^{1}$ corresponds with the dynamics of $\mathbf{X}$ in the neighborhood of the infinity. Since $\mathbb{S}^{2}$ is a curved surface, for working with the vector field $p(\mathbf{X})$ on $\mathbb{S}^{2}$, we need the expressions of this vector field in the local charts $\left(U_{i}, \phi_{i}\right)$ and $\left(V_{i}, \psi_{i}\right)$, where $U_{i}=\left\{y \in \mathbb{S}^{2}: y_{i}>0\right\}, V_{i}=\left\{y \in \mathbb{S}^{2}: y_{i}<0\right\}, \phi_{i}: U_{i} \longrightarrow \mathbb{R}^{2}$ and $\psi_{i}: V_{i} \longrightarrow \mathbb{R}^{2}$ for $i=1,2,3$, with $\phi_{i}(y)=-\psi_{i}(y)=\left(y_{m} / y_{i}, y_{n} / y_{i}\right)$ for $m<n$ and $m, n \neq i$. In the local chart $\left(U_{1}, \phi_{1}\right)$ the expression of $p(\mathbf{X})$ is

$$
\dot{u}=v^{d}\left[-u P\left(\frac{1}{v}, \frac{u}{v}\right)+Q\left(\frac{1}{v}, \frac{u}{v}\right)\right], \quad \dot{v}=-v^{d+1} P\left(\frac{1}{v}, \frac{u}{v}\right) .
$$

In $\left(U_{2}, \phi_{2}\right)$ the expression of $p(\mathbf{X})$ is

$$
\dot{u}=v^{d}\left[P\left(\frac{u}{v}, \frac{1}{v}\right)-u Q\left(\frac{u}{v}, \frac{1}{v}\right)\right], \quad \dot{v}=-v^{d+1} Q\left(\frac{u}{v}, \frac{1}{v}\right),
$$

and for $\left(U_{3}, \phi_{3}\right)$ is

$$
\dot{u}=P(u, v), \quad \dot{v}=Q(u, v) .
$$

The expressions for $p(\mathbf{X})$ in the local chart $\left(V_{i}, \psi_{i}\right)$ is the same than in the local chart $\left(U_{i}, \phi_{i}\right)$ multiplied by $(-1)^{d-1}$ for $i=1,2,3$. The points of $\mathbb{S}^{1}$ in any local chart have its $v$ coordinate equal to zero.

We note that the equator $\mathbb{S}^{1}$ is invariant by the vector field $p(\mathbf{X})$. The infinite singular points of $\mathbf{X}$ are the singular points of $p(\mathbf{X})$ which lie in $\mathbb{S}^{1}$. Note that if $y \in \mathbb{S}^{1}$ is an infinite singular point, then $-y$ is also an infinite singular point and these two points have the same (respectively opposite) stability if the degree of vector field is odd (respectively even).

The image of the northern hemisphere of $\mathbb{S}^{2}$ onto the plane $y_{3}=0$ under the projection $\pi\left(y_{1}, y_{2}, y_{3}\right)=\left(y_{1}, y_{2}\right)$ is called the Poincaré disc which is denoted by $\mathbb{D}$. The integral curves of $\mathbb{S}^{2}$ are symmetric with respect to the origin, therefore it is sufficient to investigate the flow of $p(\mathbf{X})$ only in the closed northern hemisphere. In order to draw the phase portrait on the Poincaré disc it is needed to project by $\pi$ the phase portrait of $p(\mathbf{X})$ on the northern hemisphere of $\mathbb{S}^{2}$. 
We note that the points $(u, 0)$ are the points of the infinity in the local charts $U_{i}$ and $V_{i}$ with $i=1,2$. Moreover, we remark that for studying the infinite singularities it is sufficient to study them on the local chart $U_{1}$, and to check if the origin of the local chart $U_{2}$ is or not a singularity.

\subsection{Topological equivalence of two polynomial vector fields.} Let $\mathbf{X}_{1}$ and $\mathbf{X}_{2}$ be two polynomial vector fields on $\mathbb{R}^{2}$. We say that they are topologically equivalent if there exists a homeomorphism on the Poincaré disc $\mathbb{D}$ which preserves the infinity $\mathbb{S}^{1}$ and sends the orbits of $\pi\left(p\left(\mathbf{X}_{1}\right)\right)$ to orbits of $\pi\left(p\left(\mathbf{X}_{2}\right)\right)$, preserving or reversing the orientation of all the orbits.

A separatrix of the Poincaré compactification $\pi(p(\mathbf{X}))$ is one of following orbits: all the orbits at the infinity $\mathbb{S}^{1}$, the finite singular points, the limit cycles, and the two orbits at the boundary of a hyperbolic sector at a finite or an infinite singular point, see for more details on the separatrices $[6,8]$.

The set of all separatrices of $\pi(p(\mathbf{X}))$, which we denote by $\Sigma_{\mathbf{X}}$, is a closed set (see [8]).

A canonical region of $\pi(p(\mathbf{X}))$ is an open connected component of $\mathbb{D} \backslash \Sigma_{\mathbf{X}}$. The union of the set $\Sigma_{\mathbf{X}}$ with an orbit of each canonical region form the separatrix configuration of $\pi(p(\mathbf{X}))$ and is denoted by $\Sigma_{\mathbf{X}}^{\prime}$. We denote the number of separatrices of a phase portrait in the Poincaré disc by $S$, and its number of canonical regions by $R$.

Two separatrix configurations $\Sigma_{\mathbf{X}_{1}}^{\prime}$ and $\Sigma_{\mathbf{X}_{2}}^{\prime}$ are topologically equivalent if there is a homeomorphism $h: \mathbb{D} \longrightarrow \mathbb{D}$ such that $h\left(\Sigma_{\mathbf{X}_{1}}^{\prime}\right)=\Sigma_{\mathbf{X}_{2}}^{\prime}$.

According to the following theorem which was proved by Markus [6], Neumann [8] and Peixoto [9], it is sufficient to investigate the separatrix configuration of a polynomial differential system, for determining its global phase portrait.

Theorem 5. Two Poincaré compactified polynomial vector fields $\pi\left(p\left(\mathbf{X}_{1}\right)\right)$ and $\pi\left(p\left(\mathbf{X}_{2}\right)\right)$ with finitely many separatrices are topologically equivalent if and only if their separatrix configurations $\Sigma_{\mathbf{X}_{1}}^{\prime}$ and $\Sigma_{\mathbf{X}_{2}}^{\prime}$ are topologically equivalent.

\section{Proof of Theorem 1}

It is easy to see that all singular points of system (2) have coordinates $(x, 0)$ where $x$ is a root of the equation $f(x)=-x+\prod_{i=1}^{k}\left(x^{2}-r_{i}^{2}\right)=0$. 
First we shall show that the equation $f(x)=0$ for all $k$ has a root in the interval $\left(-r_{1}, r_{1}\right)$. Since $f(0)=(-1)^{k} \prod_{i=1}^{k} r_{i}^{2}$, if $k$ is even then $f(0)>0$, and if $k$ is odd then $f(0)<0$. Moreover, $f\left(r_{1}\right)=-r_{1}<0$ and $f\left(-r_{1}\right)=r_{1}>0$, therefore if $k$ is even then $f(0) f\left(r_{1}\right)<0$ and if $k$ is odd then $f(0) f\left(-r_{1}\right)<0$, and these imply that the function $f(x)$ for all $k$ has at least one zero in the interval $\left(-r_{1}, r_{1}\right)$.

If $k$ is even and $x \in\left(0, r_{1}\right)$, then $f(x)$ is strictly decreasing and, if $x \in$ $\left(-r_{1}, 0\right)$ then $f(x)$ is positive. Hence for $k$ even, the equation $f(x)=0$ has only one root in the interval $\left(-r_{1}, r_{1}\right)$. By similar argument, we can easily show that if $k$ is odd, then the equation $f(x)=0$ has exactly one root in the interval $\left(-r_{1}, r_{1}\right)$. Thus system (2) has a unique singular point inside the circle $x^{2}+y^{2}=r_{1}^{2}$.

The Jacobian matrix of the system at any singular point $(x, 0)$ is as follows

$$
M=\left(\begin{array}{ll}
0 & 1 \\
f^{\prime}(x) & 0
\end{array}\right)
$$

with

$$
\operatorname{tr}(M)=0, \quad \operatorname{det}(M)=-f^{\prime}(x)=1-\sum_{j=1}^{k} 2 x \prod_{i=1, i \neq j}^{k}\left(x^{2}-r_{i}^{2}\right),
$$

tr and det represent the trace and determinant of a matrix, respectively. Let $(x, 0)$ be the singular point inside the disc of radius $r_{1}$. If $k$ is even, then $x \in\left(0, r_{1}\right)$ and $\operatorname{det}(M)>0$. If $k$ is odd, then $x \in\left(-r_{1}, 0\right)$ and $\operatorname{det}(M)>0$. Hence the singular point $(x, 0)$ is either a focus or a center, because its eigenvalues are purely imaginary. Since system (2) has a first integral defined in the interior of the disc of radius $r_{1}$, it follows that the singular point $(x, 0)$ is a center. This completes the proof of Theorem 1.

\section{Global phase portrait of system (2)}

4.1. Infinite singular points. Here we study the infinite singular points of system (2) for all $k$. The Poincaré compactification of system (2) in local chart $\left(U_{1}, \phi_{1}\right)$ is

$$
\dot{u}=-v^{2 k-1}\left(1+u^{2}\right)+\prod_{i=1}^{k}\left(1+u^{2}-r_{i}^{2} v^{2}\right), \quad \dot{v}=-v^{2 k} u .
$$

It is obvious that there is no singular point in this local chart. 


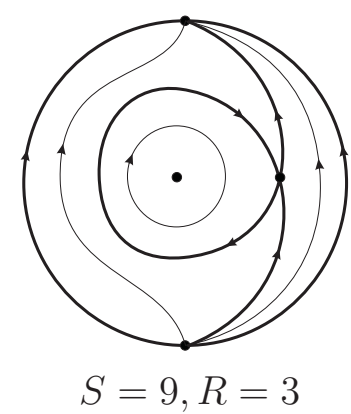

Figure 1. The phase portrait in the Poincaré disc of system (2) for $k=1$.

The expression for $p(\mathbf{X})$ in the local chart $\left(U_{2}, \phi_{2}\right)$ has the form

$$
\begin{aligned}
& \dot{u}=v^{2 k-1}\left(1+u^{2}\right)-u \prod_{i=1}^{k}\left(1+u^{2}-r_{i}^{2} v^{2}\right), \\
& \dot{v}=v^{2 k} u-v \prod_{i=1}^{k}\left(1+u^{2}-r_{i}^{2} v^{2}\right) .
\end{aligned}
$$

So the unique infinite singular point in $U_{2}$ is the origin which is a hyperbolic stable node. Since the degree of system (2) is even, the origin of the chart $V_{2}$ is a hyperbolic unstable node.

4.2. Proofs of Theorems $\mathbf{2}, \mathbf{3}$ and $\mathbf{4}$. In general system (2) has two important properties that we use for drawing its phase portrait. These properties are:

(i) Since system (2) has the inverse integrating factor (3), its corresponding first integral is defined in the whole plane except perhaps on the circles $x^{2}+y^{2}=r_{i}^{2}$. Therefore system (2) cannot have any focus as a singular point.

(ii) System (2) is invariant by the change $(x, y, t) \mapsto(x,-y,-t)$. Thus, the phase portrait of this system is symmetric with respect to the $x$-axis.

Proof of Theorem 2. System (2) with $k=1$ has the two finite singular points $P_{ \pm}=\left(\frac{1 \pm \sqrt{1+4 r_{1}^{2}}}{2}, 0\right)$. The Jacobian matrix at the point $P_{ \pm}$is

$$
M_{ \pm}=\left(\begin{array}{cc}
0 & 1 \\
\pm \sqrt{1+4 r_{1}^{2}} & 0
\end{array}\right)
$$




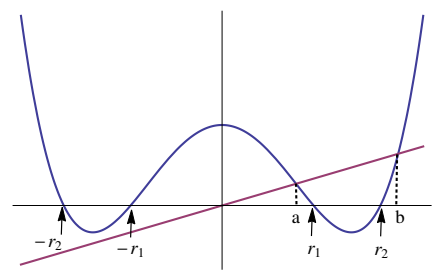

(a)

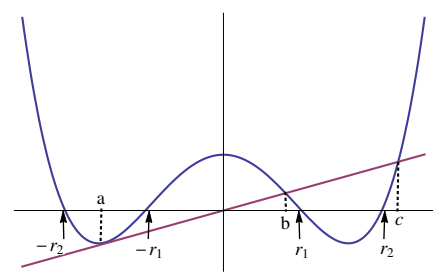

(b)

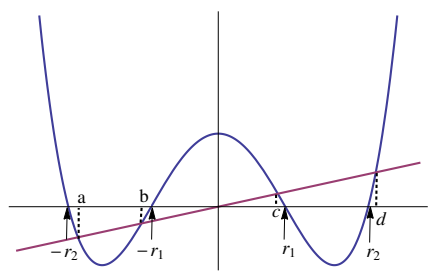

(c)

Figure 2. The graphics for all different cases of fixed points of $g(x)$ when $k=2$.

Therefore $P_{+}$is a hyperbolic saddle, and $P_{-}$is a center.

By using the symmetry $(x, y, t) \mapsto(x,-y,-t)$, the first integral (4), and the result of subsection 4.1, it follows that the global phase portrait of system (2) for $k=1$ in the Poincaré disc is topologically equivalent to the phase portrait of Figure 1.

Proof of Theorem 3. For finding the finite singular points $(x, y)$ of system (2) with $k=2$, we must take $y=0$, and find the real zeros of the equation $f(x)=-x+\left(x^{2}-r_{1}^{2}\right)\left(x^{2}-r_{2}^{2}\right)=0$. In other words, it is enough to find the fixed points of the polynomial $g(x)=\left(x^{2}-r_{1}^{2}\right)\left(x^{2}-r_{2}^{2}\right)$. Since $g(0)=r_{1}^{2} r_{2}^{2}>0$ and function $g$ has four real roots $\pm r_{1}, \pm r_{2}$, we have exactly one of the following three cases:

(i) $f$ has two simple positive roots, see Figure 2(a).

(ii) $f$ has one double negative and two simple positive roots, see Figure 2(b).

(iii) $f$ has two simple negative and two simple positive roots, see Figure 2(c).

The Jacobian matrix at every singular point $(x, 0)$ of system $(2)$ with $k=2$ is

$$
M=\left(\begin{array}{ll}
0 & 1 \\
4 x^{3}-2 x\left(r_{1}^{2}+r_{2}^{2}\right)-1 & 0
\end{array}\right)
$$




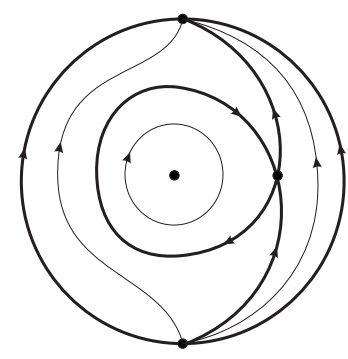

(a) $S=9, R=3$

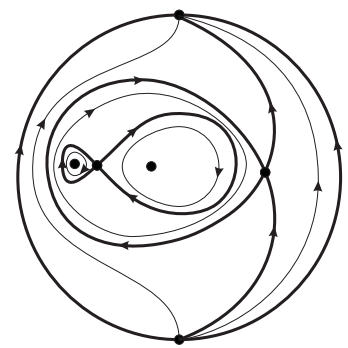

(c) $S=13, R=5$

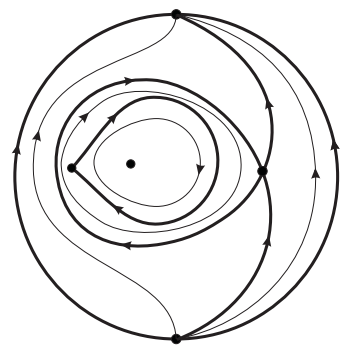

(b) $S=11, R=4$

Figure 3. The phase portraits in the Poincaré disc of system (2) for $k=2$

It is easy to see that $\operatorname{tr} M=0$ and $\operatorname{det} M=-f^{\prime}(x)$.

In case $(i)$ the polynomial $f(x)$ has only two simple positive roots $x=a$ and $x=b$ satisfying $0<a<r_{1}<r_{2}<b,-f^{\prime}(a)>0$ and $-f^{\prime}(b)<0$, see Figure 2(a). Therefore in this case system (2) with $k=2$ has two singular points $(a, 0)$ and $(b, 0)$, which are a center and a hyperbolic saddle, respectively.

For case $(i i)$ the polynomial $f(x)$ has one negative double root $x=a$, and two simple positive roots $x=b$ and $x=c$, where $-r_{2}<a<-r_{1}<$ $0<b<r_{1}<r_{2}<c,-f^{\prime}(a)=0,-f^{\prime}(b)>0$ and $-f^{\prime}(c)<0$, see Figure 2(b). Thus in this case system (2) with $k=2$ has three singular points $(a, 0),(b, 0)$ and $(c, 0)$, where $(a, 0)$ is a nilpotent singular point, and $(b, 0)$ and $(c, 0)$ are a center and a hyperbolic saddle, respectively. Here for determining the local phase portrait of the nilpotent singular point $(a, 0)$, we use the index theory. Based on the Poincaré-Hopf theorem, for every vector field on $\mathbb{S}^{2}$ with finitely many singular points, the sum of their (topological) indices is two, see for instance [2]. By applying this theorem to the Poincaré sphere with the Poincaré compactification of our system, it is easy to see that the index of the singular point 
$(a, 0)$ is zero. Since the flow of a Hamiltonian system preserve the area, and the unique nilpotent singular points with index zero are the saddle-nodes and the cusps (see Theorem 3.5 of [2]), it follows that the singular point $(a, 0)$ is a cusp.

In case (iii) the polynomial $f(x)$ has two simple negative roots $x=a$ and $x=b$, and two simple positive roots $x=c$ and $x=d$, where $-r_{2}<a<b<-r_{1}<0<c<r_{1}<r_{2}<d,-f^{\prime}(a)>0,-f^{\prime}(b)<0$, $-f^{\prime}(c)>0$ and $-f^{\prime}(d)<0$, see Figure 2(c). Therefore in this case system (2) with $k=2$ has four singular points $(a, 0),(b, 0),(c, 0)$ and $(d, 0)$, where $(a, 0)$ and $(c, 0)$ are centers, and $(b, 0)$ and $(d, 0)$ are hyperbolic saddles.

Hence, by using the symmetry $(x, y, t) \mapsto(x,-y,-t)$, the first integral (5), and that at infinity we have a pair of nodes at the origins of the local charts $U_{2}$ and $V_{2}$, the first stable and the second unstable (see subsection 4.1), it follows that the global phase portrait of system (2) for $k=2$ for each of three cases $(i),($ ii $)$ and (iii) in the Poincaré disc is topologically equivalent to the one of the phase portrait (a), (b) and (c) of Figure 3, respectively.

Proof of Theorem 4. In a similar to the proof of Theorem 3, for finding the finite singular points $(x, y)$ of system $(2)$ with $k=3$, we must have $y=0$ and $x$ must be a real zero of the equation $f(x)=-x+\left(x^{2}-\right.$ $\left.r_{1}^{2}\right)\left(x^{2}-r_{2}^{2}\right)\left(x^{2}-r_{3}^{2}\right)=0$. Hence, it is enough to find the fixed points of the polynomial function $g(x)=\left(x^{2}-r_{1}^{2}\right)\left(x^{2}-r_{2}^{2}\right)\left(x^{2}-r_{3}^{2}\right)$. Since $g(0)=-r_{1}^{2} r_{2}^{2} r_{3}^{2}<0$ and the polynomial $g(x)$ has six real roots $\pm r_{1}, \pm r_{2}$ and $\pm r_{3}$, we have exactly one of the following nine cases for the roots of the polynomial $f(x)$.

(i) One simple negative and one simple positive roots, see Figure $4(\mathrm{a})$.

(ii) One simple negative, one double positive and one simple positive roots, see Figure 4(b).

(iii) One simple negative and three simple positive roots, see Figure $4(c)$.

(iv) Three simple negative and three simple positive roots, see Figure $4(\mathrm{~d})$.

$(v)$ Three simple negative and one simple positive roots, see Figure $4(\mathrm{e})$.

(vi) One double negative, one simple negative and one simple positive roots, see Figure 4(f). 


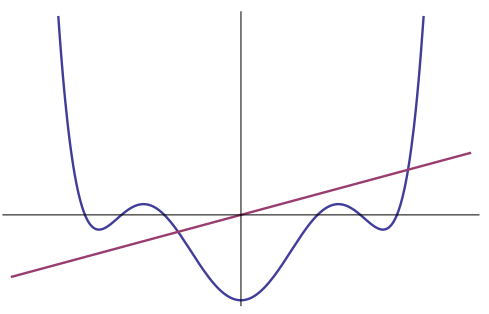

(a)

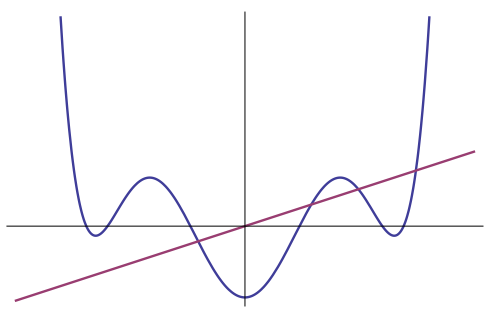

(c)

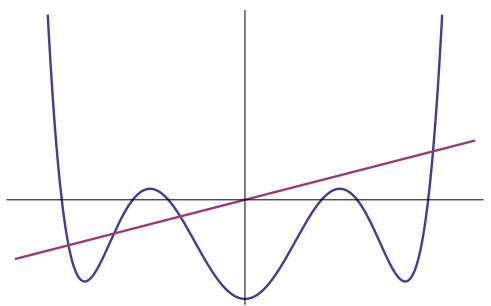

(e)

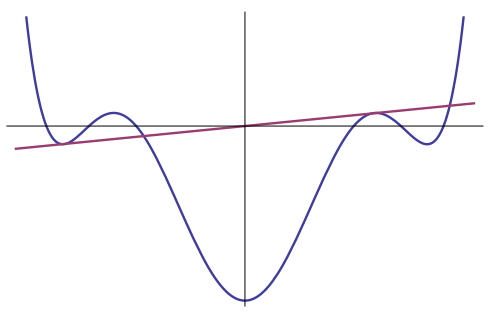

(g)

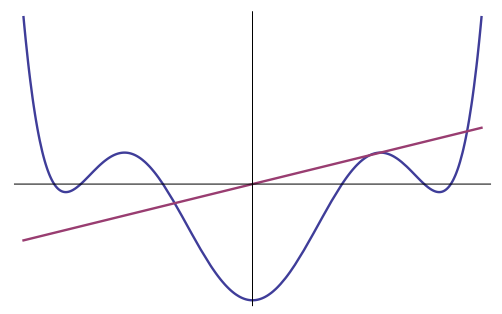

(b)

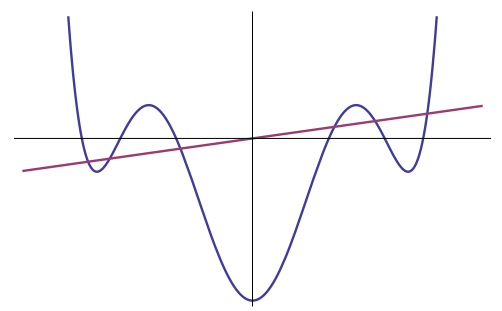

(d)

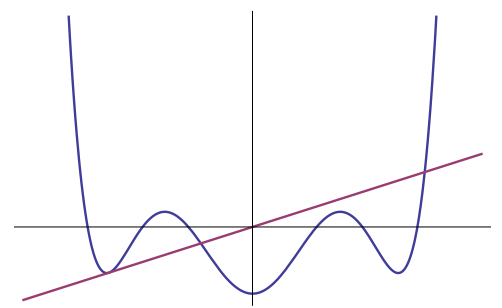

(f)

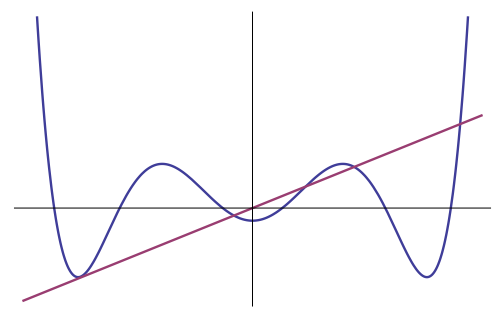

(h)

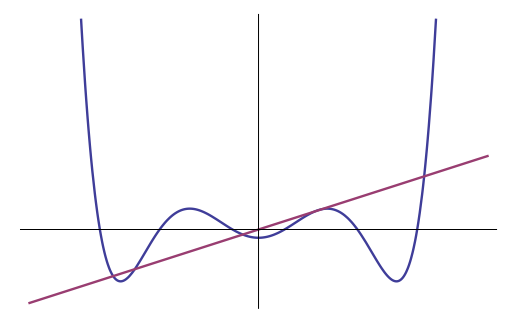

(i)

Figure 4. The graphics for all different cases of fixed points of $g(x)$ when $k=3$. 
(vii) One double negative, one simple negative, one double positive and one simple positive roots, see Figure $4(\mathrm{~g})$.

(viii) One double negative, one simple negative and three simple positive roots, see Figure 4(h).

(ix) Three simple negative, one double positive and one simple positive roots, see Figure 4(i).

The three invariant algebraic curves $x^{2}+y^{2}=r_{i}^{2}$ for $i=1,2,3$, play an important role in drawing the phase portraits for system (2) with $k=3$. Actually, if there is one singular point inside and one singular point outside of an invariant algebraic curve, then these two singular points do not have any connection, i.e. there are no orbits going from one to the other.

Case $(i)$ : In this case we have two singular points $(a, 0)$ and $(b, 0)$ where $-r_{3}<-r_{2}<-r_{1}<a<0<r_{1}<r_{2}<r_{3}<b$. By computing the Jacobian matrix in each singular point, we can conclude that $(a, 0)$ is a center and $(b, 0)$ is a hyperbolic saddle. The symmetry $(x, y, t) \mapsto(x,-y,-t)$ and the first integral (6) together with the result of subsection 4.1 force to the system to have a phase portrait topologically equivalent to the phase portrait of Figure 5(a).

Case (ii): Then system (2) has three singular points $(a, 0),(b, 0)$ and $(c, 0)$, where $-r_{3}<-r_{2}<-r_{1}<a<0<r_{1}<b<r_{2}<r_{3}<c$. Again, by computing the Jacobian matrix in each singular point we have $(a, 0)$ is a center and $(c, 0)$ is a hyperbolic saddle. Using the index theory as it is done in case $(i i)$ for $k=2$, we can conclude that $(b, 0)$ is a nilpotent cusp. Since the cusp $(b, 0)$ is the only singular point between the two invariant algebraic curves $x^{2}+y^{2}=r_{1}^{2}$ and $x^{2}+y^{2}=r_{2}^{2}$, it implies the existence of a cuspidal loop which surrounds the center $(a, 0)$ and the invariant algebraic curve $x^{2}+y^{2}=r_{1}^{2}$. By using the symmetry $(x, y, t) \mapsto(x,-y,-t)$ and the first integral (6), we also obtain a homoclinic loop passing through $(c, 0)$ and surrounding all the finite singular points and all the three invariant algebraic curves. By taking into account the result of subsection 4.1, the phase portrait of system (2) in this case is topologically equivalent to the phase portrait of Figure 5(b).

Case (iii): Then the system has four singular points $(a, 0),(b, 0)$, $(c, 0)$ and $(d, 0)$, where $-r_{3}<-r_{2}<-r_{1}<a<0<r_{1}<b<$ $c<r_{2}<r_{3}<d$. By computing the Jacobian matrix in each of these singular points, we have that $(a, 0)$ and $(c, 0)$ are centers, and $(b, 0)$ and $(d, 0)$ are hyperbolic saddles. Due to the fact that the singular point $(b, 0)$ is located between the two centers $(a, 0)$ and $(c, 0)$, and inside 

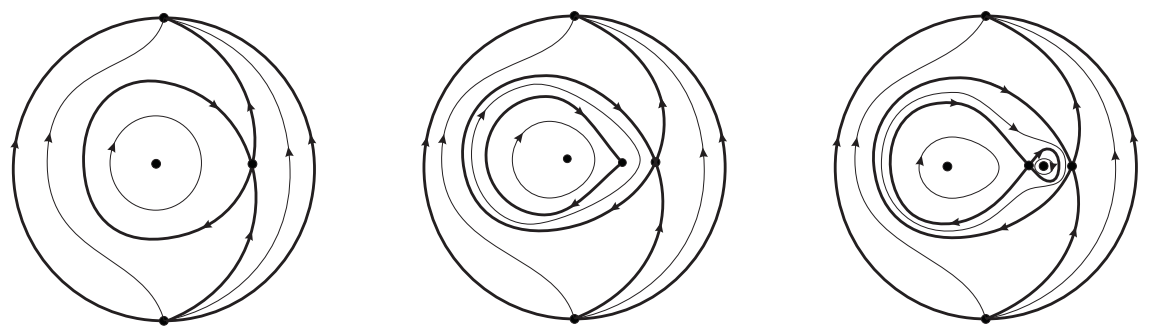

(a) $S=9, R=3$

(b) $S=11, R=4$

(c) $S=13, R=5$
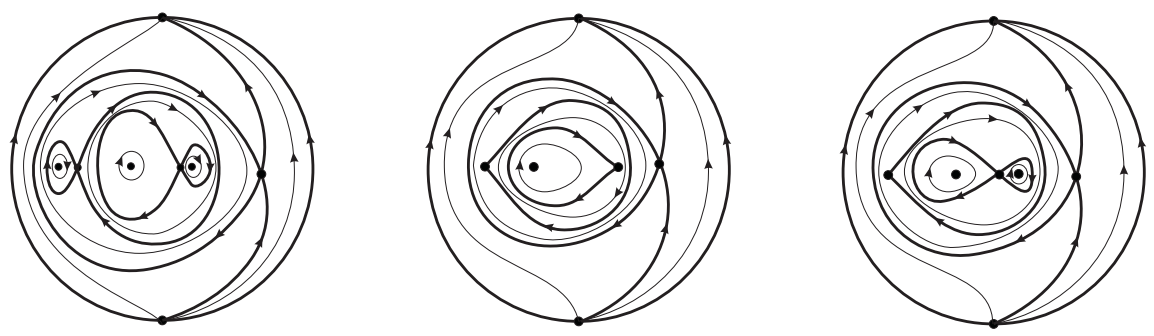

(d) $S=17, R=7$

(e) $S=13, R=5$

(f) $S=15, R=6$

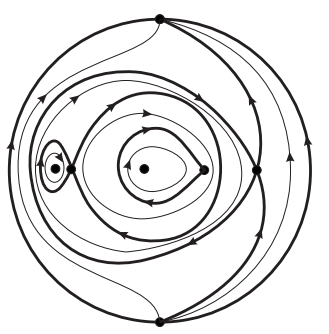

(g) $S=15, R=6$

Figure 5. The global phase portraits in the Poincaré disc of system (2) for $k=3$

the invariant algebraic curve $x^{2}+y^{2}=r_{2}^{2}$, the point $(b, 0)$ is a saddle having two homoclinic loops. The left homoclinic loop surrounds the center $(a, 0)$ and the invariant algebraic curve $x^{2}+y^{2}=r_{1}^{2}$ and the right one surrounds the center $(c, 0)$. The point $(d, 0)$ is a hyperbolic saddle on the right side of $r_{3}$. Two of its separatrics form a homoclinic loop which surrounds the other three singular points and the three algebraic invariant circles. The symmetry $(x, y, t) \mapsto(x,-y,-t)$, the first integral (6) and the result of subsection 4.1 show that the phase portrait of system (2) in this case is topologically equivalent to the phase portrait of Figure 5(c). 
Case (iv): We have six singular points $(a, 0),(b, 0),(c, 0),(d, 0)$, $(e, 0)$ and $(f, 0)$, where $-r_{3}<a<b<-r_{2}<-r_{1}<c<0<r_{1}<$ $d<e<r_{2}<r_{3}<f$. The singular points $(a, 0),(c, 0)$ and $(e, 0)$ are centers and $(b, 0),(d, 0)$ and $(f, 0)$ are hyperbolic saddles. With a similar discussion as to the one of the previous case we obtain that the phase portrait of the system is topologically equivalent to the phase portrait of Figure 5(d).

Case $(v)$ : Doing a similar discussion to the case (iii), we obtain that the phase portrait of the system is topologically equivalent to the phase portrait of Figure 5(c).

Case (vi): Working in a similar way to the case (ii), we obtain that the phase portrait of the system is topologically equivalent to the phase portrait of Figure 5(b).

Case (vii): We have four singular points $(a, 0),(b, 0),(c, 0)$ and $(d, 0)$, where $-r_{3}<a<-r_{2}<-r_{1}<b<0<r_{1}<c<r_{2}<r_{3}<d$. By obtaining the Jacobian matrix in each singular point we get that $(b, 0)$ is a center and $(d, 0)$ is a hyperbolic saddle. By using the index theory and Corollary 2 in chapter 3 of [10], it follows that the singular point $(b, 0)$ inside the invariant algebraic curve $x^{2}+y^{2}=r_{1}^{2}$, and the singular point $(a, 0)$ inside the invariant algebraic curve $x^{2}+y^{2}=r_{3}^{2}$, are cusps. Using the invariant algebraic curves together with the symmetry $(x, y, t) \mapsto(x,-y,-t)$ and the first integral (6) we obtain that the phase portrait of the system is topologically equivalent to the phase portrait of Figure 5(e).

Case (viii): Again in this case using similar arguments to previous cases we conclude that there are five singular points $(a, 0),(b, 0),(c, 0)$, $(d, 0)$ and $(e, 0)$, where $-r_{3}<a<-r_{2}<-r_{1}<b<0<r_{1}<c<d<$ $r_{2}<r_{3}<e$. The singular points $(b, 0)$ and $(d, 0)$ are centers, $(e, 0)$ and $(c, 0)$ are hyperbolic saddles and $(a, 0)$ is a cusp. In this case the phase portrait is topologically equivalent to the one of Figure $5(\mathrm{f})$.

Case $(i x)$ : The phase portrait of system (2) in this case is topologically equivalent to the phase portrait that it is shown in Figure $5(\mathrm{~g})$. Similar to the case (viii), we have five singular points $(a, 0),(b, 0)$, $(c, 0),(d, 0)$ and $(e, 0)$, where $-r_{3}<a<b<-r_{2}<-r_{1}<c<0<$ $r_{1}<d<r_{2}<r_{3}<e$. The singular points $(a, 0)$ and $(c, 0)$ are centers, $(b, 0)$ and $(e, 0)$ are hyperbolic saddles and $(d, 0)$ is a cusp.

This completes the proof of Theorem 4 . 


\section{ACKNOWLEDGEMENTS}

This work is supported by the Ministerio de Economìa, Industria y competitividad, Agencia Estatal de Investigación grant MTM201677278-P (FEDER), the Agència de Gestió d'Ajusts Universitaris i de Recerca grant 2017SGR1617, and the European project DynamicsH2020-MSCA-RISE-2017-777911.

\section{REFERENCES}

[1] H. Dulac, Détermination et integration dune certaine classe déquations différentielle ayant par point singulier un centre, Bull. Sci. Math. Sér. (2) 32 (1908), 230-252.

[2] F. Dumortier, J. Llibre And J.C. Artés, Qualitative Theory of Planar Differential Systems, Springer Verlag, New York, 2006.

[3] H. Giacomini, J. Gine And J. Llibre, The problem of distinguishing between a center and a focus for nilpotent and degenerate analytic systems, J. Diff. Eqs. 227 (2006), 406-426.

[4] M. Grau And J. Llibre, Divergence and Poincaré Liapunov constants for analytic differential systems, J. Diff. Eqs. 258 (2015), 4348-4367.

[5] J. Llibre And H. Zoladek, The Poincare center problem, J. Dyn Control Syst. 14 (2008), 505-535.

[6] L. Markus, Global structure of ordinary differential equations in the plane: Trans. Amer. Math Soc. 76 (1954), 127-148.

[7] L. Mazzi and M. Sabatini, A characterization of centres via first integrals, J. Diff. Eqs. 76 (1988), 222-237.

[8] D. A. Neumann, Classification of continuous flows on 2-manifolds, Proc. Amer. Math. Soc. 48 (1975), 73-81.

[9] M.M. Peixoto, On the classification of flows on 2-manifolds. Academic, New York, pages 389-419, 1973. Dynamical systems (Proc. Sympos., Univ. Bahia, Salvador, 1971).

[10] L. Perko, Differential Equations and Dynamical Systems, 3rd Ed, Springer, 2001.

[11] H. Poincaré, Mémoire sur les courbes définies par les équations différentielles, Journal de Mathématiques 37 (1881), 375-422; Oeuvres de Henri Poincaré, vol. I, Gauthier-Villars, Paris, 1951, pp 3-84.

${ }^{1}$ Departament de Matemàtiques, Universitat Autònoma de Barcelona, 08193 Bellaterra, Barcelona, Catalonia, Spain

E-mail address: jllibre@mat.uab.cat

Department of Mathematical Sciences, Isfahan University of TechNOLOGY, ISFAHAN, IRAN, 84156-83111.

E-mail address: marzieh.mousavi@math.iut.ac.ir

E-mail address: a.nabavi@math.iut.ac.ir 